\title{
PROJETO DE EXTENSÃO UNIVERSITÁRIA: UM ESPAÇO PROPICIO PARA A FORMAÇÃO DO PROFISSIONAL DE ENGENHARIA
}

Ana Manoela de Castro Santos - eng.manoelacastro@gmail.com

Unidade de Ensino Superior de Feira de Santana

Av. Eduardo Magalhães Subaé

44.079-002 - Feira de Santana - Bahia

Resumo: O presente estudo objetiva a abordagem dos aspectos teóricos e práticos que envolvem o projeto de extensão universitária da Unidade de Ensino Superior de Feira de Santana (UNEF) intitulado "UNEF Sustentável". O projeto em questão tem por objetivo proporcionar aos discentes dos vários cursos da instituição aprendizagens e vivências em diversos campos da promoção da sustentabilidade em empresas de grande porte, permitindo um intercâmbio entre os cursos da mesma. Para analisar a percepção dos participantes deste projeto foi elaborado um questionário semiaberto com 10 questões relacionadas com facilidades e dificuldades enfrentadas no desenvolvimento das pesquisas e sobre a importância da extensão na vida profissional. Foram entrevistados 20 dos monitores do projeto, onde todos são voluntários, a maior dificuldade foi a exposição do trabalho ao público, já a maior facilidade foi o trabalho em equipe. Todos os entrevistados apontaram que a maior contribuição pessoal obtida por meio de Projeto de Extensão foi melhorar a comunicação com o público, ademais os pesquisadores deram destaque a importância da extensão universitária para o desenvolvimento profissional e da sociedade.

Palavras-chave: Projeto de Extensão; Extensão Universitária; Contribuição social.

\section{INTRODUÇÃO}

Segundo o Plano Nacional de Extensão Universitária (PNEX, 2001) a extensão universitária é o processo educativo, cultural e científico que promove de forma associada o ensino e a pesquisa de determinado tópico, promovendo uma relação transformadora entre Instituições de Ensino e a Sociedade.

Para Serrano (2013) ao se pensar nas Instituições de Ensino a partir do seu objetivo básico, a formação do profissional, vê-se que este é um processo complexo devido a diversidade do trabalho acadêmico. Baseado nisso, a extensão universitária tem sua importância na apresentação de uma diversidade conceitual e prática sobre o objeto de estudo, permitindo a geração de conhecimento e desenvolvimento de novas habilidades.

Segundo Tumelero (2018) o projeto de extensão representa um dos três pilares das universidades de graduação, juntamente com o ensino e a pesquisa. Tumelero destaca a importância da participação em um projeto na hora de formular um currículo, pois eles ampliam a atuação do campus universitário para além das salas de aula, articulando de maneira prática o conhecimento científico, do ensino e da pesquisa, com as necessidades da comunidade onde a Instituição se insere, interagindo e transformando a sociedade.

A Lei $n^{\circ} 9.394$ (Brasil, 1996) trata sobre as diretrizes e bases de educação nacional, citando a prática da Extensão Universitária, considerando-a como atividade acadêmica que busca a articulação do Ensino e da Pesquisa, relacionando a Universidade e a Sociedade criando então uma relação autônoma e crítico-positiva da extensão, por meio de programas estruturantes que permitam o desenvolvimento social. 
Silva e Vasconcelos (2006) afirmam que no Brasil a educação superior prioriza o ensino e a pesquisa, mas não aborda de maneira abrangente as atividades de extensão consideradas por ele indispensáveis para a formação profissional.

Considerando as atividades de extensão universitária fundamentais para a formação completar pessoal e profissional, dando enfoque aos estudantes de engenharia, surge a motivação para esta pesquisa. Têm-se, então, o objetivo de avaliar a percepção dos pesquisadores da contribuição da extensão universitária na formação dos estudantes dos cursos de Engenharia Civil, Elétrica, Mecânica e Química da Unidade de Ensino Superior de Feira de Santana (UNEF).

\section{MATERIAIS E MÉTODOS}

O público alvo deste estudo foram os estudantes dos cursos de Engenharia Civil, Elétrica, Mecânica e Química participantes do projeto de extensão "UNEF Sustentável”, em suas variadas áreas de aplicação, que desenvolveram atividades de educação em sustentabilidade por no mínimo 12 (doze) meses.

O Projeto "UNEF Sustentável" realiza atividades de desenvolvimento de projetos e ações para a educação ambiental dentro da própria Instituição, tais como: Seminários de educação ambiental, desenvolvimento de Plano de Gerenciamento de Resíduos Sólidos (PGRS), desenvolvimento de projetos para reuso de água e substituição do uso da energia convencional por energia solar, dentre outros.

Visando analisar a percepção dos estudantes participantes do Projeto de Extensão, foi elaborado um questionário semiaberto com 10 (dez) perguntas relacionadas as dificuldades e facilidades enfrentadas durante o desenvolvimento da pesquisa, e as opiniões individuais sobre a importância da participação no projeto para a vida profissional e pessoal de cada um dos envolvidos.

A coleta de dados foi realizada entre os meses de abril e maio de 2020, sendo este questionário enviado aos participantes ativos e egressos do projeto via email, em sequência os dados foram processados com o auxílio da ferramenta Excel, apresentando percentagens por meio de gráficos, possibilitando uma melhor visualização dos resultados obtidos.

\section{RESULTADOS E DISCUSSÕES}

Foram entrevistados 20 pesquisadores do Projeto de Extensão "UNEF Sustentável", sendo todos voluntários o que demonstra maior interesse pela vivência prática em detrimento da remuneração. Os resultados obtidos foram elencados de forma a estarem organizados nos seguintes tópicos: tempo de participação dos monitores, área de enfoque na pesquisa, dificuldades na extensão, facilidades na extensão, desenvolvimento acadêmico e desenvolvimento pessoal.

\subsection{Tempo de participação dos monitores no projeto de extensão}

O tempo de participação dos entrevistados foi de 1 a 2 anos, que acaba por ser o tempo de existência do Projeto. Para Serrano (2013) este número é considerado bom ou além da expectativa de permanência, outro fator positivo se dá pelo voluntarismo de todos os entrevistados, pois, para ele, este é o início de uma tomada de consciência da necessidade de mudanças na forma de atuação das Instituições em relação com a sociedade, a Figura 1 relaciona os dados obtidos por meio de um gráfico. 


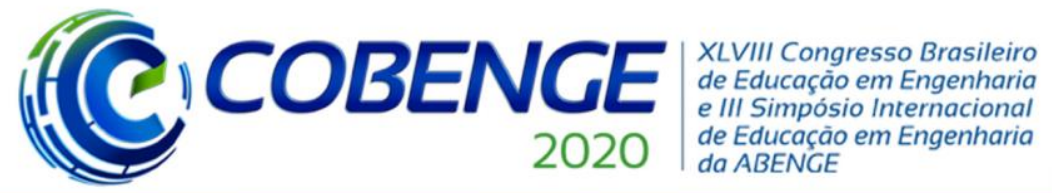

"Os desafios para formar hoje o engenheiro do amanhã"

Figura 1 - Tempo de participação dos pesquisadores no Projeto

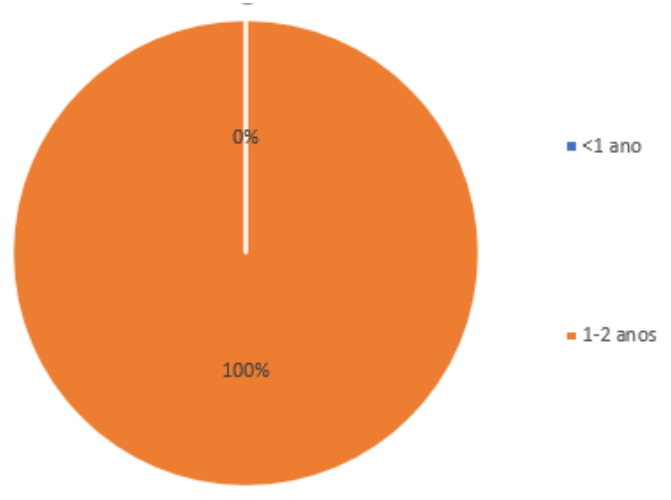

Fonte: Autor (2020)

\section{2 Área de enfoque no projeto de extensão}

O Projeto "UNEF Sustentável" possui 3 (três) áreas principais de estudo, sendo elas: Gestão de Resíduos Sólidos, Reuso de Água e Implementação de Energia Solar, buscando desenvolver projetos e ações sociais voltadas para a educação e conscientização sobre essas temáticas, a Figura 2 representa as áreas de pesquisa dos entrevistados.

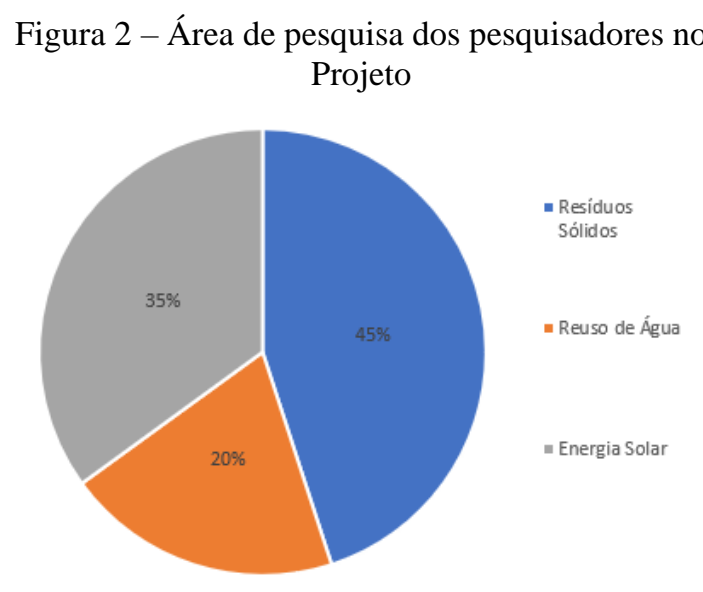

Fonte: Autor (2020)

\subsection{Dificuldades encontradas na extensão}

A maior parte dos entrevistados respondeu que a maior dificuldade encontrada durante o desenvolvimento do Projeto de Extensão foi a "exposição do trabalho ao público" (37\%) seguida por "busca por referências relacionadas ao tema" $(25 \%)$. Outros tópicos foram abordados, porém em menor escala, como "realização de eventos educacionais" (17\%), "trabalho em equipe" (13\%) e "elaboração e realização de dinâmicas" $(8 \%)$, nenhum dos entrevistados mencionou "não possuir dificuldades", como é possível visualizar na Figura 3. 


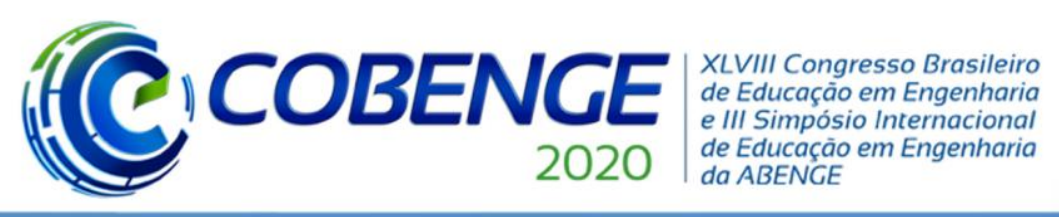

"Os desafios para formar hoje o engenheiro do amanhã"

Figura 3 - Dificuldades dos pesquisadores no Projeto

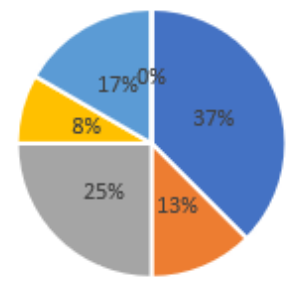

$$
\begin{aligned}
& \text { - Exposição do } \\
& \text { trabalho } \\
& \text { - Trabalho em } \\
& \text { equipe } \\
& \text { - Busca por } \\
& \text { referências } \\
& \text { - Elaboração de } \\
& \text { dinâmicas } \\
& \text { - Realização de } \\
& \text { eventos } \\
& \text { - Não possui }
\end{aligned}
$$

Fonte: Autor (2020)

Cerca de $92 \%$ dos entrevistados aponta ter superado suas dificuldades, $4 \%$ não superaram e $4 \%$ não responderam. Os entrevistados que superaram suas dificuldades apontam que o fizeram durante o período de participação do Projeto, o que reforça a afirmação de Ribeiro (2005) que diz que a dificuldade é um aprendizado e reforça os dados obtidos por Biondi e Alves (2011).

As dificuldades que surgem durante a realização de um trabalho de extensão podem se apresentar de diversas formas como, por exemplo, obstáculos que impedem a efetivação do projeto, ou podem proporcionar aprendizado e um incentivo ao estudo mais aprofundado de determinado tema.

\subsection{Facilidades encontradas na extensão}

Cerca de $40 \%$ dos entrevistados apontou que a maior facilidade foi o "Trabalho em Equipe", seguido da "Elaboração do Projeto" (24\%). Outros tópicos como "Uso de softwares" (8\%), "Tomada de decisões" (16\%) e "Domínio de conceitos técnicos" (12\%) apresentaram percentuais menores, entretanto não desprezíveis como se observa na Figura 4.

Figura 4 - Facilidades dos pesquisadores no Projeto

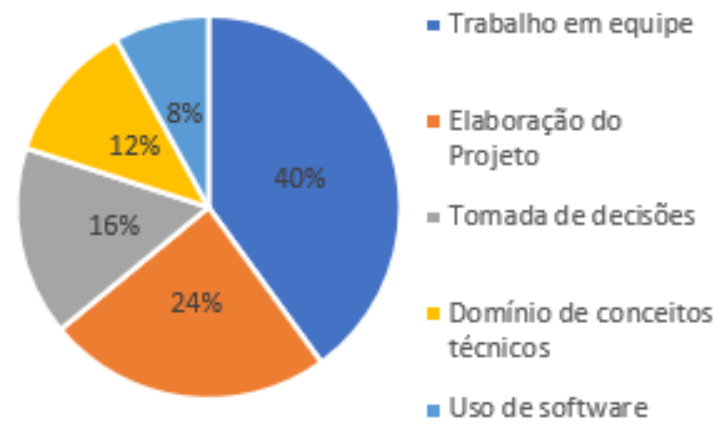

\subsection{Desenvolvimento profissional/acadêmico}

Por meio da Figura 5 é possível observar que as maiores contribuições da extensão no desenvolvimento profissional/acadêmico dos entrevistados foram na realização de atividades que não estão presentes na grade curricular do curso, desenvolvimento de projeto de extensão 
(C) COBENGE

"Os desafios para formar hoje o engenheiro do amanhã"
O1 a 03 de dezembro Evento On-line

e desenvolvimento de projeto de pesquisa, todos com $26 \%$. Este resultado aponta para a preocupação do estudante em participar de vivências práticas que agreguem no perfil técnico do profissional, buscando solucionar individualmente as possíveis deficiências de cada curso.

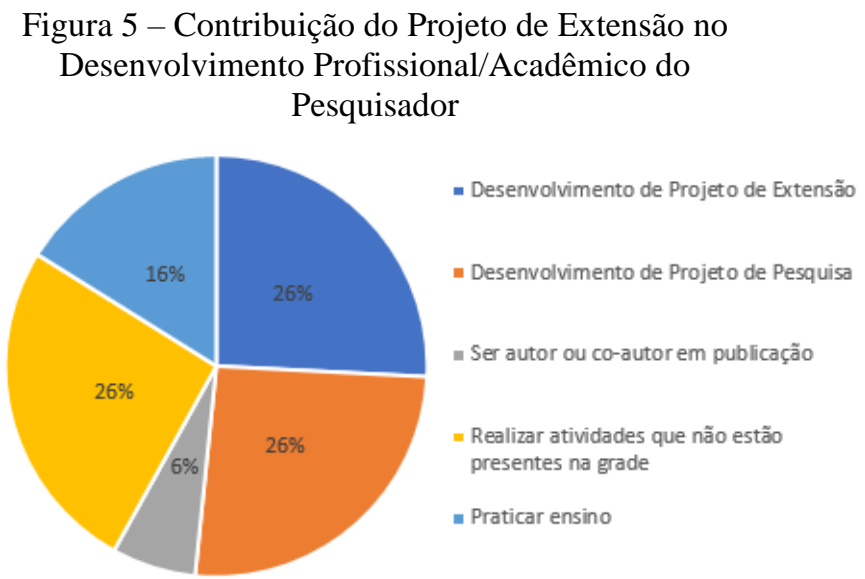

Fonte: Autor (2020)

Para Silva e Vasconcelos (2006) a inserção da extensão em um curso precisa de uma flexibilização da estrutura curricular que possibilite o envolvimento dos estudantes no mesmo, permitindo que as atividades desenvolvidas possam ser creditadas como componente curricular. $\mathrm{O}$ mesmo sugere um modelo que inserisse a Extensão desde o início do curso até o final, tornando-a um eixo de formação contínuo.

\subsection{Desenvolvimento pessoal}

Os entrevistados apontaram "Ter mais segurança" como a maior contribuição pessoal, com 35\%, os demais resultados se mostraram equilibrados como mostra a Figura 6. Sendo assim a Extensão se mostra de grande valia na formação de recursos humanos de forma completa, profissional/acadêmico e pessoal (BIONDI E ALVES, 2011).

Figura 6 - Contribuição do Projeto de Extensão no

Desenvolvimento Pessoal do Pesquisador

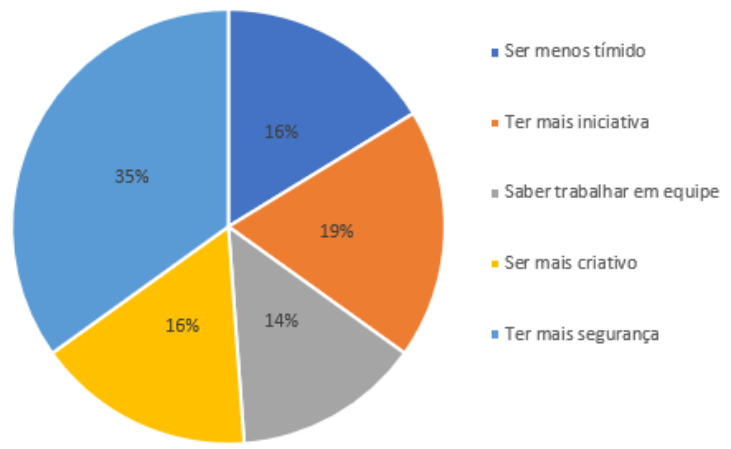

Fonte: Autor (2020)

Para Felipe (2020) o papel da Extensão Universitária é ampliar a visão de mundo de todos os envolvidos nas atividades. O mesmo ainda destaque que é por meio da prática 
extensionista que a educação assume um caráter de transformação. Entretanto nas Instituições de Ensino brasileiras ainda são poucos os estudantes que vêem a importância da extensão universitária para a formação profissional e pessoal (BIONDI E ALVES, 2011).

Por meio da extensão universitária a comunidade acadêmica tem a possibilidade de elaborar e vivenciar conhecimentos práticos, implementando-os e gerando impactos sociais positivos. Martins (2008) destaca que a promoção do conhecimento por meio da conscientização das realidades vivenciadas e a compreensão do papel individual enquanto cidadão e enquanto profissional propicia uma visão mais globalizada do conhecimento.

\section{CONCLUSÃO}

Todos os pesquisadores entrevistados participam/participaram do projeto por período igual ou superior a 1 ano, sendo grande parte deles atuantes na área de "Gestão de Resíduos Sólidos". A dificuldade encontrada mais citada foi a "exposição do trabalho ao público", já a maior facilidade foi o "trabalho em equipe", quanto a contribuição da participação do Projeto no desenvolvimento profissional/acadêmico tem-se a "realização de atividades de atividades que não estão na grade", "desenvolvimento de projeto de extensão" e "desenvolvimento de projeto de pesquisa" como principais pontos, sendo a maior contribuição no âmbito pessoal "ter mais segurança".

Por meio da pesquisa foi possível avaliar a importância da Extensão Universitária em diversos âmbitos como nos cursos de Engenharia da Instituição, na vida profissional/acadêmica e pessoal dos pesquisadores envolvidos. Os resultados demonstram os impactos positivos da vivência da extensão na formação dos estudantes, deixando evidente os benefícios já adquiridos com a participação dos mesmos.

\section{Agradecimentos}

A autora agradece todo apoio fornecido pela Unidade de Ensino Superior de Feira de Santana (UNEF) em destaque ao Prof. Andersson Cardeiro e a Prof. ${ }^{\text {a }}$ Maria das Neves, orientadores do Projeto de Extensão UNEF Sustentável. Gratidão ao Mestres Thiago Pacheco, Daliana Possari e Paulo Vitor por todo incentivo no desenvolvimento profissional. Meu carinho ao Eng $^{\circ}$ Felipe Albuquerque, obrigada por toda paciência ao longo dessa trajetória.

\section{REFERÊNCIAS}

BIONDI, Daniela; ALVES, Gabriela Cardozo. A extensão universitária na formação de estudantes do curso de Engenharia Florestal-UFPR. REMEA-Revista Eletrônica do Mestrado em Educação Ambiental, v. 26, 2011.

BRASIL. Lei $\mathrm{n}^{\circ}$ 9.394, de 20 de dezembro de 1996. Estabelece as diretrizes e bases da educação nacional. 1996

EXTENSÃO UNIVERSITÁRIA: organização e sistematização / Fórum de Pró-Reitores de Extensão das Universidades Públicas Brasileiras; organização: Edison José Corrêa. Coordenação Nacional do FORPROEX. - Belo Horizonte: Coopmed, 2007. 112p.

FELLIPE, W. C. Aprender a ser ao aprender a fazer. Disponível em: http://www1.pucminas.br/proex/arquivos/wanderley_chieppe_felippe_151.pdf. Acesso em 23 de maio 2020. 
FÓRUM DE PRÓ-REITORES DE EXTENSÃO DAS UNIVERSIDADES PÚBLICAS BRASILEIRAS. Plano Nacional de Extensão (1999-2001). Brasília. SESU/MEC, 1999.

MARTINS, L. M. Ensino-pesquisa-extensão como fundamento metodológico da construção do conhecimento na universidade. Disponível em: http://www.franca.unesp.br/oep/Eixo\%202\%20-\%20Tema\%203.pdf. Acesso em 24 de maio de 2020.

RAUBER, S. B. Extensão universitária e formação profissional indissociáveis no processo de aprendizagem da Universidade Católica de Brasília. Disponível em:

http://www.pucpr.br/eventos/educere/educere2008/anais/pdf/792_883.pdf. Acesso em 23 de maio de 2020.

RIBEIRO, K. S. Q. S. A contribuição da extensão comunitária para a formação acadêmica em fisioterapia. Fisioterapia e Pesquisa, v.12, n.3, p.22-29, 2005.

SERRANO, R. M. S. M. Conceitos de extensão universitária: um diálogo com Paulo Freire. Grupo de Pesquisa em Extensão Popular, v. 13, n. 8, 2013.

SILVA, M. S.; VASCONCELOS, S. D. Extensão universitária e formação profissional: avaliação da experiência das Ciências Biológicas na Universidade Federal de Pernambuco. Estudos em Avaliação Educacional, v.17, n.33, p.119-134, 2006.

TUMELERO, N. Projeto de extensão universitária: definições, como criar e participar. Disponível em: https://blog.mettzer.com/projeto-de-extensao-na-universidade/ Acessado em 24 de maio de 2020. 


\title{
INSTRUCTIONS FOR PREPARATION AND SUBMISSION OF WORKS TO THE SCIENTIFIC COMMITTEE OF XLVI BRAZILIAN CONGRESS OF ENGINEERING EDUCATION
}

\begin{abstract}
The present study aims to approach the theoretical and practical aspects that one involves the university extension project of the Higher Education Unit of Feira de Santana (UNEF) entitled "UNEF Sustentável". The project in question aims to provide students of the institution's various courses with learning and experiences in various fields of sustainability promotion in large companies, allowing an exchange between the courses of the same. To analyze the perception of the participants in this project, a semi-open questionnaire was elaborated with 10 questions related to facilities and difficulties faced in the development of research and about the importance of extension in professional life. 20 of the project's monitors were interviewed, where all are volunteers, the greatest difficulty was the exposure of the work to the public, while the greatest facility was teamwork. All respondents pointed out that the greatest personal contribution obtained through the Extension Project was to improve communication with the public, in addition, the researchers highlighted the importance of university extension for professional and society development.
\end{abstract}

Keywords: Extension project; University Extension; Social contribution. 